\title{
Inactivation of pancreatic digestive proteases by deconjugated bilirubin from the liver: A critical mechanism for gut protection
}

\author{
Xiaofa Qin* \\ GI Biopharma Inc, 918 Willow Grove Road, Westfield, NJ 07090, USA
}

Received: February 21, 2016; Accepted: April 01, 2016; Published: May 02 , 2016

*Corresponding author: Xiaofa Qin, GI Biopharma Inc, 918 Willow Grove Road, Westfield, NJ 07090, USA, Tel: 908-463-7423; Email: xiaofa_qin@yahoo.com

Each day, a large amount of different kinds of digestive enzymes are produced by the pancreas and discharged into the gut to digest the food $[1,2]$. Among these enzymes, those such as amylase are made in the pancreas as the active form. However, digestive enzymes such as trypsin and chymotrypsin are initially created in pancreas in the form of non-active zymogens as trypsinogen and chymotrypsinogen, which are only activated in the lumen of gut where the digestion of food occurred. This would have reflected the high potential of self-damage they may strike, as the protein of the body are just the same by nature as those from the meat or egg. However, up to date, the exact profound mechanism as how the gut is protected from the damage by these pancreatic digestive proteases remained poorly investigated and noticed. Here I address that inactivation of digestive proteases by the deconjugated bilirubin would have played a critical role.

As we know, in human and many other animals, bilirubin is excreted as the end product of heme originated mainly from the senescent red blood cells $[3,4]$. The formation of bilirubin from heme is a two-step process [3]. First, heme is transformed to biliverdin by heme oxygenase. Then biliverdin is converted to bilirubin by biliverdin reductase. Intriguingly, in some animals, biliverdin has been the end product of heme [4]. It is well documented that biliverdin predominant species tends to be herbivores such as rabbits, nutrias (rodents eating water plants), sloths (leave eater), some birds like chicken, duck, and geese, or some fish such as tilapia (a fish eating algae), while bilirubin predominant species tends to be carnivores or omnivores such as cats, dogs, opossums, armadillos, alligators, African clawed toads, bullfrogs, mudpuppies, sharks (spiney dogfish), small skates, trout, goosefish, and perch [4,5], regardless the stage of evolution. Although cattle and sheep are herbivores and their bile consists mainly of bilirubin, the activity of their hepatic biliverdin reductase was just $4-5 \%$ of that of the rats [6], and their bile indeed contains certain amounts of biliverdin [4], suggesting they might be just in the intermediary state of transition.

I found pancreatic digestive proteases can be inactivated by unconjugated free bilirubin but not conjugated bilirubin or biliverdin, suggesting inactivation of digestive proteases and thus protecting the gut may be the evolutionary driving force for bilirubin or biliverdin predominance in animals [7].

Bilirubin is secreted from the bile to the lumen mainly in the conjugated form [4]. Thus, the digestion of dietary proteins in the upper small intestine would be preceded smoothly. As the epithelial cells of the gut contain certain amounts $\beta$-glucuronidase, the enzyme that catalyzes deconjugation of bilirubin [8], thus deconjugation of biliary bilirubin would occurred at the surface of the gut to form a protective layer against damage by the pancreatic digestive proteases in gut lumen. Nevertheless, large amounts of $\beta$-glucuronidase exists in gut bacteria [8], which would further result in the prompt inactivation of pancreatic digestive proteases in the lower gut. Here we can see a wonderful design of nature that turns a waste byproduct into a precious treasure.

Accumulating evidences indicate that impairment in this process may lead to devastating consequences. Evidences I collected during the last fifteen years suggested that impairment in the bilirubin deconjugation mediated inactivation of pancreatic digestive proteases, as the result of reduction of gut bacteria along with the improved hygiene and inhibition by dietary chemicals such as saccharin and sucralose may have played a critical role in the pathogenesis of inflammatory bowel disease (IBD) including ulcerative colitis and Crohn's disease [9-11]. This is also in accordance with the fact that patients with primary sclerosing cholangitis, a disease with impaired release of bile from the liver, are accompanied by high rates of IBD [12]. Nevertheless, damage of gut barrier by the poorly inactivated digestive proteases and increased infiltration of bacterial toxicants may have played an important role in a variety of other autoimmune, allergic, metabolic, neurologic diseases such as asthma, multiple sclerosis, diabetes, obesity, autism, and even cancer [13]. Evidence also suggests that increased infiltration of pancreatic digestive proteases as the result of weakening in gut barrier under critical conditions may have played critical role in the development of multiple organ dysfunction [14]. Thus inactivation of digestive 
proteases would be a likely mechanism for the effective treatment of critical patients by Calculus Bovis - the bovine gallstone that was formed mainly from the precipitated deconjugated bilirubin and had been used to treat critical patients for thousands of years in China [15].

Thus, inactivation of pancreatic digestive proteases by deconjugating bilirubin from the liver would be a critical mechanism for gut protection that demands further study.

\section{References}

1. Whitcomb DC and ME Lowe. Human pancreatic digestive enzymes. Dig Dis Sci, 2007;52(1):1-17.

2. Beck IT. The role of pancreatic enzymes in digestion. Am J Clin Nutr 1973;26(3):311-325

3. McDonagh AF. Turning green to gold. Nat Struct Biol. 2001;8(3):198200.

4. Cornelius CE. Comparative bile pigment metabolism in vertebrates, in Bile pigments and jaundice : molecular, metabolic, and medical aspects. J.D. Ostrow. 1986;601-647.

5. Cornelius CE, KC Kelley and JA Himes. Heterogeneity of bilirubin conjugates in several animal species. Cornell Veterinarian. 1975; 65(1):90-99.

6. George JW, Nulk K, Weiss A, Bruss ML, Cornelius CE. Biliverdin Reductase-Activity in Cattle, Sheep, Rabbits and Rats. International Journal of Biochemistry. 1989;21(5):477-481.
7. Qin, X. Inactivation of digestive proteases by deconjugated bilirubin: the possible evolutionary driving force for bilirubin or biliverdin predominance in animals. Gut. 2007;56(11):1641-1642.

8. Rod TO and T Midtvedt. Origin of intestinal beta-glucuronidase in germfree, monocontaminated and conventional rats. Acta Pathol Microbiol Scand [B]. 1977;85(4):271-276.

9. Qin X. Impaired inactivation of digestive proteases by deconjugated bilirubin: the possible mechanism for inflammatory bowel disease. Med Hypotheses. 2002;59(2):159-163.

10. Qin X. Etiology of inflammatory bowel disease: A unified hypothesis. World J Gastroenterol. 2012;18(15):1708-1722. doi: 10.3748/wjg. v18.i15.1708.

11. Qin X. Publications by Xiaofa Qin. [cited 2016 Feb. http://www.ncbi. nlm.nih.gov/pubmed/?term=xiaofa+qin.

12. Qin X. Primary sclerosing cholangitis and inflammatory bowel disease: where is the link? Am J Gastroenterol, 2007;102(6):1332-1333.

13. Qin X. Changes in complex microecosystem of gut and pathogenesis of diseases in modern society - Impaired inactivation of digestive proteases may be the key event. World J Complex Med. 2015;1(1):3843.

14. Altshuler AE, EB Kistler, and GW Schmid-Schonbein. Autodigestion: Proteolytic Degradation and Multiple Organ Failure in Shock. Shock. 2016;45(5):483-489. doi: 10.1097/SHK.0000000000000544.

15. Qin XF. Bilirubin would be the indispensable component for some of the most important therapeutic effects of Calculus Bovis (Niuhuang). Chin Med J (Engl). 2008;121(5):480. 